[6] C. Wey, S. Krishnan, and S. Sahli, "Design of concurrent error detectable current-mode A/D converters for real-time applications," Int. J. Analog Integrated Circuits and Signal Processing, no. 4, pp. 65-74, 1993.

[7] D. Freitas and K. Current, "CMOS current comparator circuit," Electron. Lett., vol. 19, no. 17, pp. 695-697, Aug. 1983.

[8] G. Palmisano and G. Palumbo, "High performance CMOS current comparator design," IEEE Trans. Circuits Syst. II, vol. 43, pp. 785-790, Dec. 1996.

[9] P. Crawley and G. Roberts, "High-swing MOS current mirror with arbitrarily high output resistance," Electron. Lett., vol. 28, no. 4, pp. 361-363, Feb. 1992

[10] G. Palmisano, G. Palumbo, and S. Pennisi, CMOS Current Amplifiers. New York: Academic, 1999.

[11] K. Lakshmikumar, R. Hadaway, and M. Copeland, "Characterization and modeling of mismatch in MOS transistors for precision analog design," IEEE J. Solid-State Circuits, vol. SC-21, pp. 1057-1066, Dec. 1986.

[12] M. Pelgrom, A. Duinmaijer, and A. Welbers, "Matching properties of MOS transistors," IEEE J. Solid-State Circuits, vol. 24, pp. 1433-1440, Dec. 1989.

[13] R. van de Plassche, Integrated Analog-to-Digital and Digital-to-Analog Converters. New York: Academic, 1994.

[14] Papoulis, Probability, Random Variables and Stochastic Processes, 3rd ed. New York: McGraw-Hill, 1991

\section{Anti-Jamming Performance of Chaotic Digital Communication Systems}

\author{
F. C. M. Lau, M. Ye, C. K. Tse, and S. F. Hau
}

\begin{abstract}
Among the various modulation schemes proposed for chaos-based digital communications, chaos-shift keying (CSK) and differential chaos-shift keying (DCSK) have been most thoroughly analyzed and considered for practical implementation. One important aspect of performance of any communication system is the ability to resist jamming. This area of study, however, is not available in the literature. In this paper, an analysis of antijamming performance of the coherent CSK and noncoherent DCSK systems is presented. The study includes performance analysis for the two types of systems in terms of the bit error probability under different levels of noise power, jamming power, jamming frequency and spreading factor. Computer simulations are used to verify the analytical results.
\end{abstract}

Index Terms-Anti-jamming, chaos communications, chaos-shiftkeying, differential chaos-shift-keying.

\section{INTRODUCTION}

In the past decade, chaos-based communication systems have received a great deal of attention from both the system theory and communication research communities [1]-[8]. Much of the work has focused on the basic modulation processes and the noise performance assuming ideal channel conditions. The ability to resist jamming, though is an important aspect of performance, has not been thoroughly studied. Intuitively, any coherent system, where the receiver knows or is able to reproduce the chaotic carriers, is expected to be considerably better in anti-jamming than its noncoherent counterpart. In chaos-based communication, moreover, coherent systems suffer

Manuscript received September 21, 2001; revised January 28, 2002. This work was supported in part by the Hong Kong Polytechnic University and in part by the Hong Kong Research Grants Council. This paper was recommended by Associate Editor T. Saito.

The authors are with the Department of Electronic and Information Engineering, The Hong Kong Polytechnic University, Hong Kong, China (e-mail encmlau@ @olyu.edu.hk; encktse@ polyu.edu.hk; ensfhau@ polyu.edu.hk).

Digital Object Identifier 10.1109/TCSI.2002.803362.

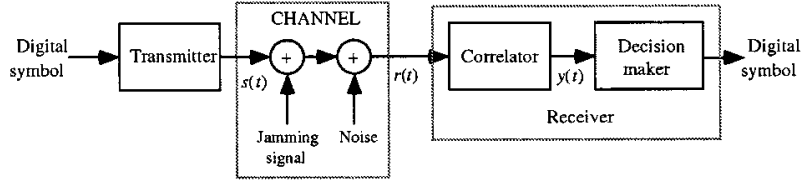

Fig. 1. Block diagram of a chaotic communication system with jamming and noise added.

serious drawbacks due to the fragility of chaos synchronization which is the main technique for reproducing the chaotic carriers [9]. Noncoherent systems are practically more favorable although their noise performance is inherently worse than coherent systems. It is, therefore, of interest to study thoroughly the antijamming properties of chaos-based communication systems in order to gain a better understanding of how well or poorly, in exact quantitative terms, a practical noncoherent chaotic communication system performs in comparison with the theoretically better coherent systems. Our purpose in this paper is to present an analytical method for evaluating anti-jamming performance of chaos-based communication systems, specifically the coherent chaos-shift-keying (CSK) system and the noncoherent differential chaos-shift-keying (DCSK) system. Analytical bit error rates (BERs) are derived, permitting evaluation of performance for a range of noise level, jamming power, jamming frequency and spreading factor. Results from computer simulations are then used to verify the analytical findings.

\section{SYSTEM OVERVIEW}

The basic problem we wish to investigate in this paper is the performance of chaos-based digital communication systems when the channel is subject to a strong jamming signal as well as additive white Gaussian noise. Fig. 1 shows a block diagram of the system under study. In our analysis, we assume that the jamming signal is a sine wave of power $P_{\mathrm{jam}}$ and frequency $f$, i.e.

$$
s_{\text {jam }}(t)=\sqrt{2 P_{\text {jam }}} \sin (2 \pi f t+\theta)
$$

where $\theta$ is an arbitrary constant phase angle. A sinusoidal jammer is investigated here because it is one of the most common jamming signals used and it can be generated easily. Suppose the transmitter output is $s(t)$. Assuming additive jamming and noise, the signal at the input of the receiver, $r(t)$, is given by

$$
r(t)=s(t)+s_{\text {jam }}(t)+\eta(t)
$$

where $\eta(t)$ is the Gaussian noise function. This signal will be used by the receiver to recover the digital symbols that have been sent to the transmitter. The method of demodulation depends of the type of the system under study. Broadly, we may differentiate two types of systems, namely coherent and noncoherent systems.

In a coherent system, the receiver has the information of the chaotic signal that carries the information [4]. This is often achieved through a so-called chaos synchronization process. Thus the detection method for a coherent system involves a correlation process operated upon the received signal and the known (reproduced) chaotic signal at the receiver. Specifically, if a few chaotic signals are used to represent some digital symbols, the correlation operation is able to differentiate accurately the different chaotic signals. Hence, a threshold detector can be used to recover the digital symbols. For a noncoherent system, however, no information about the chaotic signal is known to the receiver [10], [11]. The modulation scheme is designed such that the transmitter signal allows information to be recovered by comparing a part of the signal with another part of it.

Clearly, the coherent system should give better performance if the receiver is able to regenerate the chaotic signal and hence knows what the chaotic signal is. However, in practice (at least as known to date), 


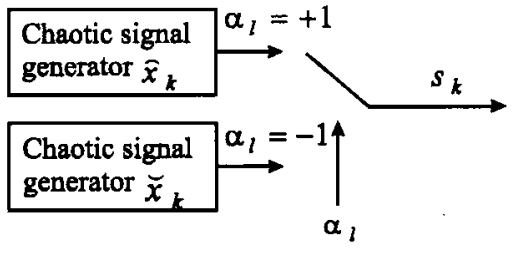

(a)

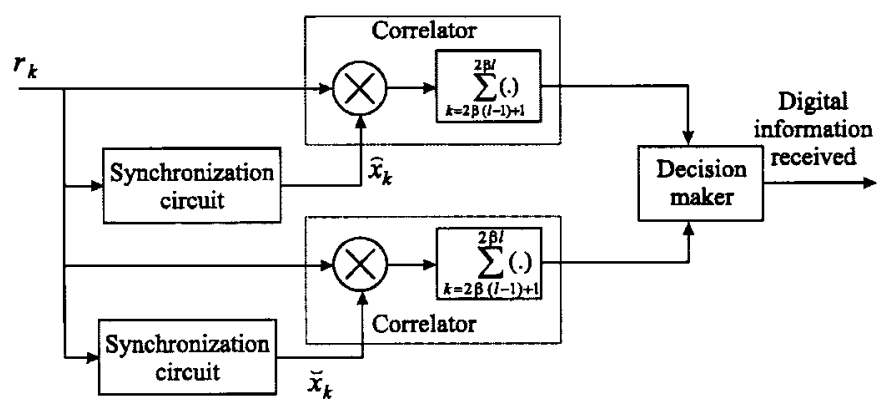

(b)

Fig. 2. Block diagram of a coherent CSK system. (a) Transmitter. (b) Receiver.

there exists no robust and reliable means of reproducing chaotic signals at the receiving end. Thus noncoherent systems still present practical and useful alternatives for chaos-based communications. In this paper we focus on the anti-jamming performance and compare the two types of systems. Specifically, in the following sections, we will consider the coherent CSK and the noncoherent DCK systems and attempt to develop analytical expressions for the bit error rates of the recovered symbols.

\section{AnAlysis of ANTi-Jamming Performance}

Instead of studying the bandpass system shown in Fig. 1 directly, we transform the bandpass system to an equivalent baseband model such that the carrier frequency of the chaotic signal is now zero [12], [13]. As a consequence, the frequency of the jammer in the bandpass model is different from its equivalent in the baseband model. Based on the equivalent model, we attempt to derive the bit error rate using discrete-time analysis. Moreover, the chaotic, jamming and noise signals are derived from different sources. Thus the assumption that they are independent of each other, which will be used throughout this paper, is well justified.

\section{A. Coherent CSK System}

We consider a discrete time binary CSK communication system, as shown in Fig. 2. In the transmitter, a pair of chaotic sequences, denoted by $\left\{\hat{x}_{k}\right\}$ and $\left\{\check{x}_{k}\right\}$, are generated by two chaotic maps. If the symbol " +1 " is sent, $\left\{\hat{x}_{k}\right\}$ is transmitted during a bit period, and if " -1 " is sent, $\left\{\check{x}_{k}\right\}$ is transmitted.

For simplicity, we consider a CSK system in which one chaos generator is used to produce chaotic signal samples $\left\{x_{k}\right\}$ for $k=1,2, \ldots$. The two possible transmitted sequences are $\left\{\hat{x}_{k}=x_{k}\right\}$ and $\left\{\breve{x}_{k}=\right.$ $\left.-x_{k}\right\}$. Suppose $\alpha_{l} \in\{-1,+1\}$ is the symbol to be sent during the $l$ th bit period and assume that " -1 " and " +1 " occur with equal probabilities. Define the spreading factor, $2 \beta$, as the number of chaotic samples used to transmit one binary symbol. During the $l$ th bit duration, i.e., for time $k=2(l-1) \beta+1,2(l-1) \beta+2, \ldots, 2 l \beta$, the output of the transmitter is

$$
s_{k}=\alpha_{l} x_{k} .
$$

In the following analysis, we assume that a jamming signal of power $P_{\mathrm{jam}}$ and frequency $f$ is added to the transmitted signal in the channel.
At the same time, white Gaussian noise is also added. The "jammed" noisy signal arriving at the receiver, denoted by $r_{k}$, is given by

$$
r_{k}=s_{k}+\sqrt{2 P_{\mathrm{jam}}} \sin \left(2 \pi k \frac{F}{2 \beta}+\theta\right)+\eta_{k}
$$

where $F$ is the normalized jamming frequency defined as

$$
F=f T_{b}
$$

and $T_{b}$ represents the bit duration, and $\theta$ is the initial phase angle of the jamming signal and is assumed to be an arbitrary constant angle selected from $[-\pi, \pi] . \eta_{k}$ is a Gaussian noise sample of zero mean and variance (power spectral density) $N_{0} / 2$. We will first consider the $l$ th bit before deriving the error probability over all transmitted bits.

Assuming that a correlator-type receiver is employed, the correlator output for the $l$ th bit $y_{l}$ is given by

$$
\begin{aligned}
y_{l}= & \sum_{k=2}^{2 \beta(l-1)+1} r_{k} x_{k} \\
= & \underbrace{\alpha_{l} \sum_{k=2 \beta(l-1)+1}^{2 \beta l} x_{k}^{2}}_{\text {required signal }}+\underbrace{\sqrt{2 P_{\mathrm{jam}}} \sum_{k=2 \beta(l-1)+1}^{2 \beta l} x_{k} \sin \left(\frac{k \pi F}{\beta}+\theta\right)}_{\text {jamming signal }} \\
& +\underbrace{\sum_{k=2 \beta(l-1)+1}^{2 \beta l} \eta_{k} x_{k}}_{\text {noise }} .
\end{aligned}
$$

Suppose a " +1 " is transmitted for the $l$ th symbol, i.e., $\alpha_{l}=+1$. For simplicity we write $y_{l} \mid\left(\alpha_{l}=+1\right)$ as

$$
y_{l} \mid\left(\alpha_{l}=+1\right)=A+B+C
$$

where $A, B$, and $C$ are the required signal, jamming signal and noise, respectively, and are defined as

$$
\begin{aligned}
& A=\sum_{k=2 \beta(l-1)+1}^{2 \beta l} x_{k}^{2} \\
& B=\sqrt{2 P_{\mathrm{jam}}} \sum_{k=2 \beta(l-1)+1}^{2 \beta l} x_{k} \sin \left(\frac{k \pi F}{\beta}+\theta\right) \\
& C=\sum_{k=2 \beta(l-1)+1}^{2 \beta l} \eta_{k} x_{k} .
\end{aligned}
$$

The mean of $y_{l} \mid\left(\alpha_{l}=+1\right)$ is

$$
\begin{aligned}
\mathrm{E}\left[y_{l} \mid\right. & \left.\left(\alpha_{l}=+1\right)\right] \\
= & \mathrm{E}[A]+\mathrm{E}[B]+\mathrm{E}[C] \\
= & \mathrm{E}\left[\sum_{k=2 \beta(l-1)+1}^{2 \beta l} x_{k}^{2}\right] \\
+ & \mathrm{E}\left[\sqrt{2 P_{\mathrm{jam}}} \sum_{k=2 \beta(l-1)+1}^{2 \beta l} x_{k} \sin \left(\frac{k \pi F}{\beta}+\theta\right)\right] \\
+ & \mathrm{E}\left[\sum_{k=2 \beta(l-1)+1}^{2 \beta l} \eta_{k} x_{k}\right] \\
= & \sum_{k=2 \beta(l-1)+1}^{2 \beta l} \mathrm{E}\left[x_{k}^{2}\right]+\sqrt{2 P_{\mathrm{jam}}} \sum_{k=2 \beta(l-1)+1}^{2 \beta l} \mathrm{E}\left[x_{k}\right] \\
& \times \mathrm{E}\left[\sin \left(\frac{k \pi F}{\beta}+\theta\right)\right]+\sum_{k=2}^{2 \beta l} \mathrm{E}\left[\eta_{k}\right] \mathrm{E}\left[x_{k}\right] \\
= & 2 \beta P_{s}+2 \beta \sqrt{2 P_{\mathrm{jam}}} \mathrm{E}\left[x_{k}\right] \mathrm{E}\left[\sin \left(\frac{k \pi F}{\beta}+\theta\right)\right]
\end{aligned}
$$


where $P_{s}=\mathrm{E}\left[x_{k}^{2}\right]$ denotes the average power of the chaotic signal. The last equality holds because $\mathrm{E}\left[\eta_{k}\right]=0$. The variance of $y_{l} \mid\left(\alpha_{l}=+1\right)$ is

$$
\begin{aligned}
\operatorname{var}\left[y_{l} \mid\left(\alpha_{l}=+1\right)\right]=\operatorname{var}[ & A]+\operatorname{var}[B]+\operatorname{var}[C]+2 \operatorname{cov}[A, B] \\
& +2 \operatorname{cov}[B, C]+2 \operatorname{cov}[A, C]
\end{aligned}
$$

where $\operatorname{cov}[X, Y]$ is the covariance between $X$ and $Y$ defined as

$$
\operatorname{cov}[X, Y]=\mathrm{E}[X Y]-\mathrm{E}[X] \mathrm{E}[Y]
$$

It can be readily shown that both $\operatorname{cov}[A, C]$ and $\operatorname{cov}[B, C]$ are zero. Hence, (12) is simplified to

$$
\operatorname{var}\left[y_{l} \mid\left(\alpha_{l}=+1\right)\right]=\operatorname{var}[A]+\operatorname{var}[B]+\operatorname{var}[C]+2 \operatorname{cov}[A, B] .
$$

The average power of the chaotic signal, denoted by $P_{s}$, can be computed by numerical simulation, or by numerical integration if the probability density function (pdf) of $\left\{x_{k}\right\}$ is available. The second term on the right hand side of (11), and the variance and covariance terms in (14) can also be computed by numerical simulation. Hence, $\mathrm{E}\left[y_{l} \mid\left(\alpha_{l}=+1\right)\right]$ and $\operatorname{var}\left[y_{l} \mid\left(\alpha_{l}=+1\right)\right]$ can be evaluated.

For the $l$ th symbol, an error occurs when $y_{l} \leq 0 \mid\left(\alpha_{l}=+1\right)$. Since $y_{l} \mid\left(\alpha_{l}=+1\right)$ is the sum of a large number of random variables, we may assume that it follows a normal distribution. The error probability is thus given by

$$
\operatorname{Prob}\left(y_{l} \leq 0 \mid\left(\alpha_{l}=+1\right)\right)=\frac{1}{2} \operatorname{erfc}\left(\frac{\mathrm{E}\left[y_{l} \mid\left(\alpha_{l}=+1\right)\right]}{\sqrt{2 \operatorname{var}\left[y_{l} \mid\left(\alpha_{l}=+1\right)\right]}}\right)
$$

where the complementary error function, $\operatorname{erfc}($.$) , is defined as$

$$
\operatorname{erfc}(\psi) \equiv \frac{2}{\sqrt{\pi}} \int_{\psi}^{\infty} e^{-\lambda^{2}} d \lambda
$$

Similarly, when a " -1 " is sent, the output of the correlator can be shown equal to

$$
y_{l} \mid\left(\alpha_{l}=-1\right)=-A+B+C .
$$

Likewise, the mean and variance of $y_{l} \mid\left(\alpha_{l}=-1\right)$, denoted by $\mathrm{E}\left[y_{l} \mid\left(\alpha_{l}=-1\right)\right]$ and $\operatorname{var}\left[y_{l} \mid\left(\alpha_{l}=-1\right)\right]$, can be derived, i.e.

$$
\begin{aligned}
\mathrm{E}\left[y_{l} \mid\left(\alpha_{l}=-1\right)\right]= & -2 \beta P_{s}+2 \beta \sqrt{2 P_{\mathrm{jam}}} \mathrm{E}\left[x_{k}\right] \\
& \times \mathrm{E}\left[\sin \left(\frac{k \pi F}{\beta}+\theta\right)\right] \\
\operatorname{var}\left[y_{l} \mid\left(\alpha_{l}=-1\right)\right]= & \operatorname{var}[A]+\operatorname{var}[B]+\operatorname{var}[C]-2 \operatorname{cov}[A, B]
\end{aligned}
$$

where $A, B$, and $C$ are defined in (8)-(10). The corresponding error probability is

$$
\operatorname{Prob}\left(y_{l}>0 \mid\left(\alpha_{l}=-1\right)\right)=\frac{1}{2} \operatorname{erfc}\left(\frac{-\mathrm{E}\left[y_{l} \mid\left(\alpha_{l}=-1\right)\right]}{\sqrt{2 \operatorname{var}\left[y_{l} \mid\left(\alpha_{l}=-1\right)\right]}}\right) .
$$

Hence, the error probability of the $l$ th transmitted symbol is

$$
\begin{aligned}
\operatorname{BER}_{\mathrm{CSK}}^{(l)}= & \operatorname{Prob}\left(\alpha_{l}=1\right) \times \operatorname{Prob}\left(y_{l} \leq 0 \mid \alpha_{l}=1\right) \\
& +\operatorname{Prob}\left(\alpha_{l}=-1\right) \times \operatorname{Prob}\left(y_{l}>0 \mid \alpha_{l}=-1\right) \\
=\frac{1}{4} & {\left[\operatorname{erfc}\left(\frac{\mathrm{E}\left[y_{l} \mid\left(\alpha_{l}=+1\right)\right]}{\sqrt{2 \operatorname{var}\left[y_{l} \mid\left(\alpha_{l}=+1\right)\right]}}\right)\right.} \\
& \left.\quad+\operatorname{erfc}\left(\frac{-\mathrm{E}\left[y_{l} \mid\left(\alpha_{l}=-1\right)\right]}{\sqrt{2 \operatorname{var}\left[y_{l} \mid\left(\alpha_{l}=-1\right)\right]}}\right)\right]
\end{aligned}
$$

Since $\mathrm{E}\left[y_{l} \mid\left(\alpha_{l}=+1\right)\right], \operatorname{var}\left[y_{l} \mid\left(\alpha_{l}=+1\right)\right], \mathrm{E}\left[y_{l} \mid\left(\alpha_{l}=-1\right)\right]$ and $\operatorname{var}\left[y_{l} \mid\left(\alpha_{l}=-1\right)\right.$ ] [given by (11), (14), (18) and (19) respectively] are all independent of $l, \mathrm{BER}_{\mathrm{CSK}}^{(l)}$ is also independent of $l$. Thus the bit error probability of the $l$ th transmitted symbol is the same as the bit error rate (BER) of the whole system. The BER of the noisy CSK system under the influence of a sinusoidal jammer, denoted by BER $_{C S K}$, is therefore

$$
\begin{aligned}
\operatorname{BER}_{\mathrm{CSK}}= & \operatorname{BER}_{\mathrm{CSK}}^{(l)} \\
= & \frac{1}{4}\left[\operatorname{erfc}\left(\frac{\mathrm{E}\left[y_{l} \mid\left(\alpha_{l}=+1\right)\right]}{\sqrt{2 \operatorname{var}\left[y_{l} \mid\left(\alpha_{l}=+1\right)\right]}}\right)\right. \\
& \left.+\operatorname{erfc}\left(\frac{-\mathrm{E}\left[y_{l} \mid\left(\alpha_{l}=-1\right)\right]}{\sqrt{2 \operatorname{var}\left[y_{l} \mid\left(\alpha_{l}=-1\right)\right]}}\right)\right] .
\end{aligned}
$$

Hence, (11), (14), (18) and (19) can be computed and substituted into (22) to obtain the BER of the system.

Further simplifications of the equations are possible if we make some assumptions.

1) The mean value of $\left\{x_{k}\right\}$ is zero. This is a reasonable assumption because it is a waste of power to send any noninformation-bearing dc component through the channel. Any dc component generated by the chaos generator can be removed artificially before transmission.

2) $\left\{x_{k}\right\}$ has a vanishing autovariance function. This can be proved to be true for some chaotic sequences.

3) $\left\{x_{k}^{2}\right\}$ has a vanishing autocovariance function.

4) The normalized frequency $F$ is an irrational number because the jamming signal and the chaotic signals are derived from different sources which are not synchronized. Therefore, we can assume that the sampled sinusoidal signal $\sin ((k \pi F / \beta)+\theta)$ is not periodic.

From the above assumptions, we have

$$
\begin{array}{rlrl}
\mathrm{E}\left[x_{k}\right] & =0 & \\
\operatorname{cov}\left[x_{j}, x_{k}\right]=\mathrm{E}\left[x_{j} x_{k}\right]-\mathrm{E}\left[x_{j}\right] \mathrm{E}\left[x_{k}\right] & =0 & & \text { for } j \neq k \\
\operatorname{cov}\left[x_{j}^{2}, x_{k}^{2}\right]=\mathrm{E}\left[x_{j}^{2} x_{k}^{2}\right]-\mathrm{E}\left[x_{j}^{2}\right] \mathrm{E}\left[x_{k}^{2}\right] & =0 & & \text { for } j \neq k \\
\mathrm{E}\left[\sin \left(\frac{k \pi F}{\beta}+\theta\right)\right] & =0 . &
\end{array}
$$

Thus (11) and (18) are simplified to

$$
\begin{aligned}
\mathrm{E}\left[y_{l} \mid\left(\alpha_{l}=+1\right)\right] & =2 \beta P_{s} \\
\mathrm{E}\left[y_{l} \mid\left(\alpha_{l}=-1\right)\right] & =-2 \beta P_{s}
\end{aligned}
$$

and the variances of the variables $A, B$ and $C$, and the covariance between $A$ and $B$ are given by

$$
\begin{aligned}
\operatorname{var}[A] & =2 \beta \Lambda \\
\operatorname{var}[B] & =2 \beta P_{\mathrm{jam}} P_{s} \\
\operatorname{var}[C] & =\beta N_{0} P_{s} \\
\operatorname{cov}[A, B] & =0
\end{aligned}
$$

where $\Lambda$ is the variance of $\left\{x_{k}^{2}\right\}$, i.e.,

$$
\Lambda=\operatorname{var}\left[x_{k}^{2}\right] .
$$

Hence, both (14) and (19) are given by

$$
\begin{aligned}
\operatorname{var}\left[y_{l} \mid\left(\alpha_{l}=+1\right)\right] & =\operatorname{var}\left[y_{l} \mid\left(\alpha_{l}=-1\right)\right] \\
& =2 \beta \Lambda+2 \beta P_{\mathrm{jam}} P_{s}+\beta N_{0} P_{s} \\
& =\beta\left(2 \Lambda+2 P_{\mathrm{jam}} P_{s}+N_{0} P_{s}\right) .
\end{aligned}
$$


Note that the larger the signal power $P_{s}$, the larger the variance of the correlator output $y_{l}$. Substituting (27), (28) and (34) into (22), the BER can be found as

$$
\begin{aligned}
\mathrm{BER}_{\mathrm{CSK}} & =\frac{1}{2} \operatorname{erfc}\left(\frac{2 \beta P_{s}}{\sqrt{2 \beta\left(2 \Lambda+2 P_{\mathrm{jam}} P_{s}+N_{0} P_{s}\right)}}\right) \\
& =\frac{1}{2} \operatorname{erfc}\left(\frac{1}{\sqrt{\frac{\Lambda}{\beta P_{s}^{2}}+\frac{P_{\mathrm{jam}}}{\beta P_{s}}+\frac{N_{0}}{2 \beta P_{s}}}}\right) \\
& =\frac{1}{2} \operatorname{erfc}\left(\frac{1}{\sqrt{\left(\frac{E_{b}^{2}}{4 \beta \Lambda}\right)^{-1}+\left(\frac{E_{b}}{2 P_{\mathrm{jam}}}\right)^{-1}+\left(\frac{E_{b}}{N_{0}}\right)^{-1}}}\right)
\end{aligned}
$$

where $E_{b}=2 \beta P_{s}$ denotes the average bit energy. The expressions given in (35) and (36) are thus the analytical bit error rate (BER) for the jammed noisy coherent CSK system. Note that for fixed jamming power $P_{\text {jam }}$ and noise power spectral density $N_{0} / 2$, the BER can be improved by making one or more of the following adjustments.

1) reduce the variance of $\left\{x_{k}^{2}\right\}$;

2) increase the spreading factor $2 \beta$;

3 ) increase the signal power $P_{s}$.

When the jamming power is zero, i.e., $P_{\text {jam }}=0$, it can be readily shown that the bit error rate reduces to

$$
\begin{aligned}
\operatorname{BER}_{\mathrm{CSK}} \mid\left(P_{\mathrm{jam}}=0\right) & =\frac{1}{2} \operatorname{erfc}\left(\frac{1}{\sqrt{\frac{\Lambda}{\beta P_{s}^{2}}+\frac{N_{0}}{2 \beta P_{s}}}}\right) \\
& =\frac{1}{2} \operatorname{erfc}\left(\frac{1}{\sqrt{\left(\frac{E_{b}^{2}}{4 \beta \Lambda}\right)^{-1}+\left(\frac{E_{b}}{N_{0}}\right)^{-1}}}\right) .
\end{aligned}
$$

Note that in the derivation of the BER, it has been assumed that an exact reference is available in the receiver. While the re-construction of the exact reference at the receiver, especially in the presence of noise and jamming, remains an unsolved problem at present, the BER derived can nonetheless provide performance benchmarks for evaluating other chaotic modulation/demodulation techniques under similar jamming conditions.

Example: Consider the case where a logistic map is used for chaos generation. The form of the map is

$$
x_{k+1}=g\left(x_{k}\right)=1-2 x_{k}^{2}
$$

and the invariant probability density function (pdf) of $\left\{x_{k}\right\}$, denoted by $\rho(x)$, is [14]

$$
\rho(x)= \begin{cases}\frac{1}{\pi \sqrt{1-x^{2}}}, & \text { if }|x|<1 \\ 0, & \text { otherwise. }\end{cases}
$$

It can be shown easily that the mean value of $\left\{x_{k}\right\}$ is zero and $\left\{x_{k}\right\}$ has a vanishing autovariance function. Also, it is proved in the Appendix that the autovariance for $\left\{x_{k}^{2}\right\}$ is vanishing for the logistic map. In other words, the first three assumptions made earlier are justified. Also, we have

$$
\begin{aligned}
& P_{s}=\mathrm{E}\left[x_{k}^{2}\right]=\int_{-\infty}^{\infty} x^{2} \rho(x) d x=\int_{-1}^{1} x^{2} \rho(x) d x=\frac{1}{2} \\
& \Lambda=\operatorname{var}\left[x_{k}^{2}\right]=\mathrm{E}\left[x_{k}^{4}\right]-\mathrm{E}^{2}\left[x_{k}^{2}\right]=\int_{-1}^{1} x^{4} \rho(x) d x-\frac{1}{4}=\frac{1}{8} .
\end{aligned}
$$

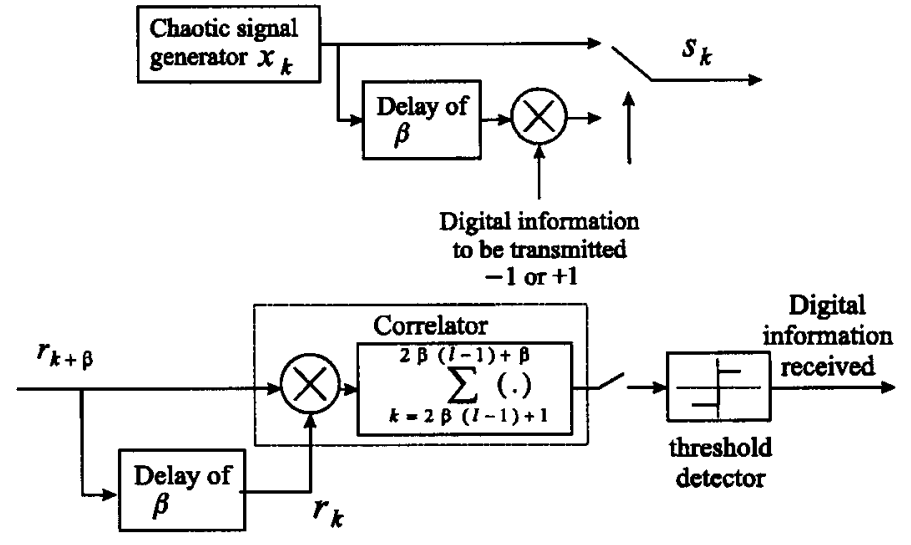

Fig. 3. Block diagram of a noncoherent DCSK system: (a) transmitter; (b) receiver.

For the case where the logistic map is used to generate the chaotic samples, we substitute (41) and (42) into (35) to obtain the BER, i.e.

$$
\mathrm{BER}_{\mathrm{CSK}}=\frac{1}{2} \operatorname{erfc}\left(\sqrt{\frac{2 \beta}{1+4 P_{\mathrm{jam}}+2 N_{0}}}\right) .
$$

\section{B. Noncoherent DCSK System}

In this section, we consider the DCSK system. The basic modulation process involves dividing the bit period into two equal slots. The first slot carries a reference chaotic signal, and the second slot bears the information. For a binary system, the second slot is the same copy or an inverted copy of the first slot depending upon the symbol sent being " +1 " or " -1 ." This structural arrangement allows the detection to be done in a noncoherent manner requiring no reproduction of the same chaotic carrying signals at the receiver. Essentially, the detection of a DCSK signal can be accomplished by correlating the first and the second slots of the same symbol and comparing the correlator output with a threshold. Fig. 3 shows the block diagrams of a DCSK transmitter and receiver pair.

As in the previous case, we assume that the signal is jammed additively by a sine wave of power $P_{\text {jam }}$ and normalized frequency $F$. In addition, the received signal contains additive white Gaussian noise. Using the same notations and functions as defined in Section III-A, the signal transmitted in the $l$ th bit can be written as

$$
s_{k}=\left\{\begin{array}{rr}
x_{k}, & \text { for } k=2 \beta(l-1)+1,2 \beta(l-1)+2, \ldots, \\
& 2 \beta(l-1)+\beta \\
\alpha_{l} x_{k-\beta}, & \text { for } k=2 \beta(l-1)+\beta+1, \\
& 2 \beta(l-1)+\beta+2, \ldots, 2 \beta l
\end{array}\right.
$$

where $\alpha_{l}$ takes on the values of \pm 1 according to the bit being sent. The noisy jammed signal, $r_{k}$, within a bit duration is given by

$$
r_{k}=s_{k}+\sqrt{2 P_{\mathrm{jam}}} \sin \left(\frac{k \pi F}{\beta}+\theta\right)+\eta_{k}
$$

where the symbols $F, \theta$ and $\eta_{k}$ are as defined previously in Section III-A. 
At the receiver, the detector essentially calculates the correlation of the reference and data slots of the same symbol. We consider the output of the correlator for the $l$ th received bit, $y_{l}$, which is given by

$$
\begin{aligned}
& y_{l}=\sum_{k=2}^{2 \beta(l-1)+\beta} r_{k} r_{k+\beta} \\
& =\sum_{k=2 \beta(l-1)+1}^{2 \beta(l-1)+\beta}\left[x_{k}+\sqrt{2 P_{\mathrm{jam}}} \sin \left(\frac{k \pi F}{\beta}+\theta\right)+\eta_{k}\right] \\
& \times\left[\alpha_{l} x_{k}+\sqrt{2 P_{\mathrm{jam}}} \sin \left(\frac{(k+\beta) \pi F}{\beta}+\theta\right)+\eta_{k+\beta}\right] \\
& =\alpha_{l} \sum_{k=2 \beta(l-1)+1}^{2 \beta(l-1)+\beta} x_{k}^{2}+\sqrt{2 P_{\mathrm{jam}}} \sum_{k=2 \beta(l-1)+1}^{2 \beta(l-1)+\beta} x_{k} \\
& \times\left[\sin \left(\frac{(k+\beta) \pi F}{\beta}+\theta\right)+\alpha_{l} \sin \left(\frac{k \pi F}{\beta}+\theta\right)\right] \\
& +2 P_{\mathrm{jam}} \sum_{k=2 \beta(l-1)+1}^{2 \beta(l-1)+\beta} \sin \left(\frac{(k+\beta) \pi F}{\beta}+\theta\right) \\
& \times \sin \left(\frac{k \pi F}{\beta}+\theta\right)+\sum_{k=2 \beta(l-1)+1}^{2 \beta(l-1)+\beta} x_{k}\left(\eta_{k+\beta}+\alpha_{l} \eta_{k}\right) \\
& +\sqrt{2 P_{\mathrm{jam}}} \sum_{k=2}^{2 \beta(l-1)+\beta}\left[\eta_{k+\beta} \sin \left(\frac{k \pi F}{\beta}+\theta\right)\right. \\
& \left.+\eta_{k} \sin \left(\frac{(k+\beta) \pi F}{\beta}+\theta\right)\right] \\
& +\sum_{k=2 \beta(l-1)+1}^{2 \beta(l-1)+\beta} \eta_{k} \eta_{k+\beta} .
\end{aligned}
$$

Define

$$
\begin{aligned}
& A=\sum_{k=2}^{2 \beta(l-1)+\beta} x_{k}^{2} \\
& B=2 \sqrt{2 P_{\mathrm{jam}}} \cos \left(\frac{\pi F}{2}\right) \sum_{k=2 \beta(l-1)+1}^{2 \beta(l-1)+\beta} x_{k} \\
& \times \sin \left(\frac{k \pi F}{\beta}+\frac{\pi F}{2}+\theta\right) \\
& B^{\prime}=2 \sqrt{2 P_{\mathrm{jam}}} \sin \left(\frac{\pi F}{2}\right) \sum_{k=2}^{2 \beta(l-1)+\beta} x_{k} \\
& \times \cos \left(\frac{k \pi F}{\beta}+\frac{\pi F}{2}+\theta\right) \\
& C=\beta P_{\mathrm{jam}} \cos (\pi F) \\
& D=\frac{-P_{\mathrm{jam}} \sin (\pi F)}{\sin (\pi F / \beta)} \cos \left(4 l \pi F-2 \pi F+\frac{\pi F}{\beta}+2 \theta\right) \\
& G=\sum_{k=2 \beta(l-1)+1}^{2 \beta(l-1)+\beta} x_{k} \eta_{k+\beta} \\
& H=\sum_{k=2 \beta(l-1)+1}^{2 \beta(l-1)+\beta} x_{k} \eta_{k}
\end{aligned}
$$

$$
\begin{aligned}
J & =\sqrt{2 P_{\text {jam }}} \sum_{k=2 \beta(l-1)+1}^{2 \beta(l-1)+\beta} \sin \left(\frac{k \pi F}{\beta}+\theta\right) \eta_{k+\beta} \\
K & =\sqrt{2 P_{\text {jam }}} \sum_{k=2 \beta(l-1)+1}^{2 \beta(l-1)+\beta} \sin \left(\frac{(k+\beta) \pi F}{\beta}+\theta\right) \eta_{k} \\
L & =\sum_{k=2 \beta(l-1)+1}^{2 \beta(l-1)+\beta} \eta_{k} \eta_{k+\beta} .
\end{aligned}
$$

Using (47)-(56), it can be easily shown that when $\alpha_{l}=+1$ and $\alpha_{l}=$ -1 , (46) becomes, respectively

$$
\begin{aligned}
& y_{l} \mid\left(\alpha_{l}=+1\right) \\
& =\underbrace{A}_{\text {required signal }}+\underbrace{B+C+D}_{\text {jamming signal }}+\underbrace{G+H+J+K+L}_{\text {noise }} \\
& y_{l} \mid\left(\alpha_{l}=-1\right) \\
& =\underbrace{-A}_{\text {required signal }}+\underbrace{B^{\prime}+C+D}_{\text {jamming signal }}+\underbrace{G-H+J+K+L}_{\text {noise }} .
\end{aligned}
$$

Similar to the case of CSK, the means and variances of $y_{l}$ given a " +1 " or " -1 " is sent can be evaluated by numerical simulations. Denote the respective means and variances by $\mathrm{E}\left[y_{l} \mid\left(\alpha_{l}= \pm 1\right)\right]$ and $\operatorname{var}\left[y_{l} \mid\left(\alpha_{l}= \pm 1\right)\right]$. As $y_{l} \mid\left(\alpha_{l}= \pm 1\right)$ is the sum of a large number of random variables, we assume that it is normally distributed. Hence, the approximated error probability of the $l$ th transmitted bit is

$$
\begin{aligned}
& \operatorname{BER}_{\mathrm{DCSK}}^{(l)}= \operatorname{Prob}\left(\alpha_{l}=1\right) \times \operatorname{Prob}\left(y_{l} \leq 0 \mid\left(\alpha_{l}=+1\right)\right) \\
&+\operatorname{Prob}\left(\alpha_{l}=-1\right) \times \operatorname{Prob}\left(y_{l}>0 \mid\left(\alpha_{l}=-1\right)\right) \\
&=\frac{1}{4}\left[\operatorname{erfc}\left(\frac{\mathrm{E}\left[y_{l} \mid\left(\alpha_{l}=+1\right)\right]}{\sqrt{2 \operatorname{var}\left[y_{l} \mid\left(\alpha_{l}=+1\right)\right]}}\right)\right. \\
&\left.\quad+\operatorname{erfc}\left(\frac{-\mathrm{E}\left[y_{l} \mid\left(\alpha_{l}=-1\right)\right]}{\sqrt{2 \operatorname{var}\left[y_{l} \mid\left(\alpha_{l}=-1\right)\right]}}\right)\right]
\end{aligned}
$$

Note that the approximation gets better with a larger spreading factor.

To simplify the analysis, we make similar assumptions as in Section III-A, i.e.:

1) the mean value of $\left\{x_{k}\right\}$ is zero;

2) $\left\{x_{k}\right\}$ has a vanishing autovariance function;

3) $\left\{x_{k}^{2}\right\}$ has a vanishing autovariance function;

4) the normalized frequency $F$ is an irrational number.

With these assumptions, it can be shown that

$$
\begin{aligned}
& \mathrm{E}\left[y_{l} \mid\left(\alpha_{l}=+1\right)\right] \\
& =\mathrm{E}[A]+\mathrm{E}[B]+\mathrm{E}[C]+\mathrm{E}[D]+\mathrm{E}[G] \\
& \quad+\mathrm{E}[H]+\mathrm{E}[J]+\mathrm{E}[K]+\mathrm{E}[L] \\
& \mathrm{E}\left[y_{l} \mid\left(\alpha_{l}=-1\right)\right] \\
& =-\mathrm{E}[A]+\mathrm{E}\left[B^{\prime}\right]+\mathrm{E}[C]+\mathrm{E}[D]+\mathrm{E}[G] \\
& \quad-\mathrm{E}[H]+\mathrm{E}[J]+\mathrm{E}[K]+\mathrm{E}[L] \\
& \operatorname{var}\left[y_{l} \mid\left(\alpha_{l}=+1\right)\right] \\
& \approx \operatorname{var}[A]+\operatorname{var}[B]+\operatorname{var}[C]+\operatorname{var}[D]+\operatorname{var}[G] \\
& \quad+\operatorname{var}[H]+\operatorname{var}[J]+\operatorname{var}[K]+\operatorname{var}[L] \\
& \operatorname{var}\left[y_{l} \mid\left(\alpha_{l}=-1\right)\right] \\
& \approx \operatorname{var}[A]+\operatorname{var}\left[B^{\prime}\right]+\operatorname{var}[C]+\operatorname{var}[D]+\operatorname{var}[G] \\
& \quad+\operatorname{var}[H]+\operatorname{var}[J]+\operatorname{var}[K]+\operatorname{var}[L] .
\end{aligned}
$$


Moreover, it can be shown readily that the relevant means and variances are

$$
\begin{array}{lc}
\mathrm{E}[A]=\beta E\left[x_{k}^{2}\right] \equiv \beta P_{s} & \operatorname{var}[A]=\beta \operatorname{var}\left[x_{k}^{2}\right] \equiv \beta \Lambda \\
\mathrm{E}[B]=0 & \operatorname{var}[B]=4 \beta P_{s} P_{\mathrm{jam}} \\
& \times \cos ^{2}\left(\frac{\pi F}{2}\right) \\
\mathrm{E}\left[B^{\prime}\right]=0 & \operatorname{var}\left[B^{\prime}\right]=4 \beta P_{s} P_{\mathrm{jam}} \\
& \times \sin ^{2}\left(\frac{\pi F}{2}\right) \\
\mathrm{E}[C]=\beta P_{\mathrm{jam}} \cos (\pi F) & \operatorname{var}[C]=0 \\
\mathrm{E}[D]=\frac{-P_{\mathrm{jam}} \sin (\pi F)}{\sin (\pi F / \beta)} & \\
\times & \\
\times \cos \left(4 l \pi F-2 \pi F+\frac{\pi F}{\beta}+2 \theta\right) & \operatorname{var}[D]=0 \\
\mathrm{E}[G]=0 & \operatorname{var}[G]=\beta P_{s} N_{0} / 2 \\
\mathrm{E}[H]=0 & \operatorname{var}[H]=\beta P_{s} N_{0} / 2 \\
\mathrm{E}[J]=0 & \operatorname{var}[J]=\beta P_{\mathrm{jam}} N_{0} / 2 \\
\mathrm{E}[K]=0 & \operatorname{var}[K]=\beta P_{\mathrm{jam}} N_{0} / 2 \\
\mathrm{E}[L]=0 & \operatorname{var}[L]=\beta N_{0}^{2} / 4 .
\end{array}
$$

Substituting (64) into (60)-(63), we obtain

$$
\begin{aligned}
\mathrm{E}\left[y_{l} \mid\left(\alpha_{l}=+1\right)\right]= & \beta P_{s}+\beta P_{\mathrm{jam}} \cos (\pi F)-\frac{P_{\mathrm{jam}} \sin (\pi F)}{\sin (\pi F / \beta)} \\
& \times \cos \left(4 l \pi F-2 \pi F+\frac{\pi F}{\beta}+2 \theta\right) \\
\mathrm{E}\left[y_{l} \mid\left(\alpha_{l}=-1\right)\right]= & -\beta P_{s}+\beta P_{\mathrm{jam}} \cos (\pi F)-\frac{P_{\mathrm{jam}} \sin (\pi F)}{\sin (\pi F / \beta)} \\
& \times \cos \left(4 l \pi F-2 \pi F+\frac{\pi F}{\beta}+2 \theta\right)
\end{aligned}
$$

$\operatorname{var}\left[y_{l} \mid\left(\alpha_{l}=+1\right)\right] \approx \beta \Lambda+4 \beta P_{s} P_{\mathrm{jam}} \cos ^{2}\left(\frac{\pi F}{2}\right)+\beta P_{s} N_{0}$

$$
+\beta P_{\mathrm{jam}} N_{0}+\frac{\beta N_{0}^{2}}{4}
$$

$$
\begin{aligned}
\operatorname{var}\left[y_{l} \mid\left(\alpha_{l}=-1\right)\right] \approx & \beta \Lambda+4 \beta P_{s} P_{\mathrm{jam}} \sin ^{2}\left(\frac{\pi F}{2}\right)+\beta P_{s} N_{0} \\
& +\beta P_{\mathrm{jam}} N_{0}+\frac{\beta N_{0}^{2}}{4} .
\end{aligned}
$$

Also, (59) now becomes (69) as shown at the bottom of the page. The average error rate over all transmitted bits is thus

$$
\mathrm{BER}_{\mathrm{DCSK}}=\lim _{N_{s} \rightarrow \infty} \frac{1}{N_{s}} \sum_{l=1}^{N_{s}} \mathrm{BER}_{\mathrm{DCSK}}^{(l)} .
$$

In (69), only the term $\cos (4 l \pi F-2 \pi F+(\pi F / \beta)+2 \theta)$ is a function of $l$. Moreover, it has been assumed that $F$ is irrational. Therefore, as $l$ varies, $4 l \pi F$ and hence $(4 l \pi F-2 \pi F+(\pi F / \beta)+2 \theta)$ will be uniformly distributed in $[-\pi,+\pi]$. Denoting $4 l \pi F-2 \pi F+(\pi F / \beta)+$ $2 \theta$ by $\gamma,(70)$ can be rewritten as

$$
\operatorname{BER}_{\text {DCSK }}=\int_{-\pi}^{+\pi} \operatorname{BER}_{\text {DCSK }}^{(l)} \nu(\gamma) d \gamma
$$

where $\nu(\gamma)=1 / 2 \pi$ is the pdf of $\gamma$. Define $W=\cos \gamma$. The pdf of $W$, denoted as $\mu(W)$, is

$$
\mu(W)= \begin{cases}\frac{1}{\pi \sqrt{1-W^{2}}}, & \text { for }|W| \leq 1 \\ 0, & \text { otherwise. }\end{cases}
$$

Thus (71) becomes (73) and (74), as shown at the bottom of the page, where $E_{b}=2 \beta P_{s}$ denotes the average bit energy. The expressions given in (73) and (74) are thus the analytical BER for the jammed noisy DCSK system. Note that for fixed jamming power $P_{\text {jam }}$ and noise power spectral density $N_{0} / 2$, the BER can be reduced by making one or a combination of the following adjustments:

1) reduce the variance of $\left\{x_{k}^{2}\right\}$;

2) increase the signal power $P_{s}$.

In each of the complimentary error functions in (73) and (74), the denominator increases with $\beta$. However, in the numerator, the variation of the terms $\beta P_{\text {jam }} \cos (\pi F)$ and $\left(P_{\text {jam }} \sin (\pi F) / \sin (\pi F / \beta)\right) W$ with $\beta$ cannot be observed easily. Therefore, the overall effect of $\beta$ on the BER performance cannot be determined simply by observing (73) or (74).

$$
\begin{aligned}
& \operatorname{BER}_{\mathrm{DCSK}}^{(l)}=\frac{1}{4}\left[\operatorname{erfc}\left(\frac{\beta P_{s}+\beta P_{\mathrm{jam}} \cos (\pi F)-\frac{P_{\mathrm{jam}} \sin (\pi F)}{\sin (\pi F / \beta)} \cos \left(4 l \pi F-2 \pi F+\frac{\pi F}{\beta}+2 \theta\right)}{\sqrt{2 \beta \Lambda+8 \beta P_{s} P_{\mathrm{jam}} \cos ^{2}\left(\frac{\pi F}{2}\right)+2 \beta P_{s} N_{0}+2 \beta P_{\mathrm{jam}} N_{0}+\frac{\beta N_{0}^{2}}{2}}}\right)\right. \\
& \left.+\operatorname{erfc}\left(\frac{\beta P_{s}-\beta P_{\mathrm{jam}} \cos (\pi F)+\frac{P_{\mathrm{jam}} \sin (\pi F)}{\sin (\pi F / \beta)} \cos \left(4 l \pi F-2 \pi F+\frac{\pi F}{\beta}+2 \theta\right)}{\sqrt{2 \beta \Lambda+8 \beta P_{s} P_{\mathrm{jam}} \sin ^{2}\left(\frac{\pi F}{2}\right)+2 \beta P_{s} N_{0}+2 \beta P_{\mathrm{jam}} N_{0}+\frac{\beta N_{0}^{2}}{2}}}\right)\right]
\end{aligned}
$$

$$
\begin{aligned}
\operatorname{BER}_{\mathrm{DCSK}}= & \int_{-1}^{+1} \operatorname{BER}_{\mathrm{DCSK}}^{(l)} \mu(W) d W \\
= & \frac{1}{4} \int_{-1}^{+1}\left[\operatorname{erfc}\left(\frac{\beta P_{s}+\beta P_{\mathrm{jam}} \cos (\pi F)-\frac{P_{\mathrm{jam}} \sin (\pi F)}{\sin (\pi F / \beta)} W}{\sqrt{2 \beta \Lambda+8 \beta P_{s} P_{\mathrm{jam}} \cos ^{2}\left(\frac{\pi F}{2}\right)+2 \beta P_{s} N_{0}+2 \beta P_{\mathrm{jam}} N_{0}+\frac{\beta N_{0}^{2}}{2}}}\right)\right. \\
& \left.+\operatorname{erfc}\left(\frac{\beta P_{s}-\beta P_{\mathrm{jam}} \cos (\pi F)+\frac{P_{\mathrm{jam}} \sin (\pi F)}{\sin (\pi F / \beta)} W}{\sqrt{2 \beta \Lambda+8 \beta P_{s} P_{\mathrm{jam}} \sin ^{2}\left(\frac{\pi F}{2}\right)+2 \beta P_{s} N_{0}+2 \beta P_{\mathrm{jam}} N_{0}+\frac{\beta N_{0}^{2}}{2}}}\right)\right] \times \frac{1}{\pi \sqrt{1-W^{2}}} d W \\
=\frac{1}{4} \int_{-1}^{+1}\left[\begin{array}{ll}
\frac{E_{b}}{2}+\beta P_{\mathrm{jam}} \cos (\pi F)-\frac{P_{\mathrm{jam}} \sin (\pi F)}{\sin (\pi F / \beta)} W \\
\operatorname{erfc}\left(\frac{E_{b}}{2}-\beta P_{\mathrm{jam}} \cos (\pi F)+\frac{P_{\mathrm{jam}} \sin (\pi F)}{\sin (\pi F / \beta)} W^{2 \beta \Lambda}\right.
\end{array}\right. & \left.\quad+\operatorname{erfc}\left(\frac{4 E_{b} P_{\mathrm{jam}} \cos ^{2}\left(\frac{\pi F}{2}\right)+E_{b} N_{0}+2 \beta P_{\mathrm{jam}} N_{0}+\frac{\beta N_{0}^{2}}{2}}{\sqrt{2 \beta \Lambda+4 E_{b} P_{\mathrm{jam}} \sin ^{2}\left(\frac{\pi F}{2}\right)+E_{b} N_{0}+2 \beta P_{\mathrm{jam}} N_{0}+\frac{\beta N_{0}^{2}}{2}}}\right)\right] \times \frac{1}{\pi \sqrt{1-W W^{2}}} d W
\end{aligned}
$$




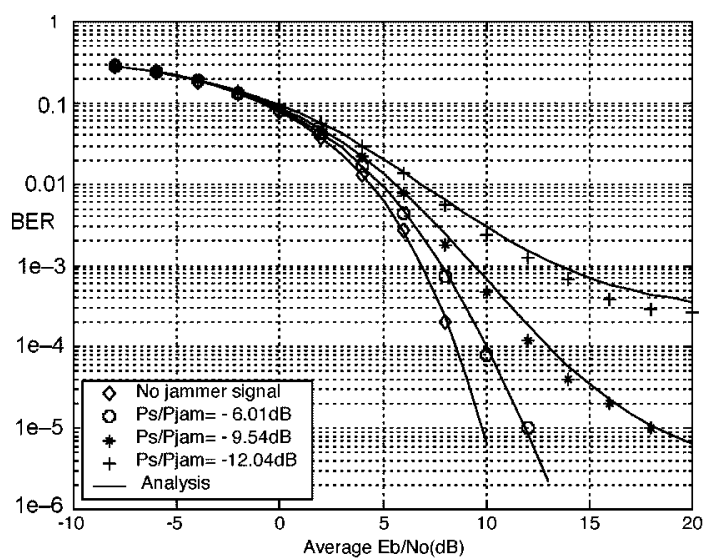

(a)

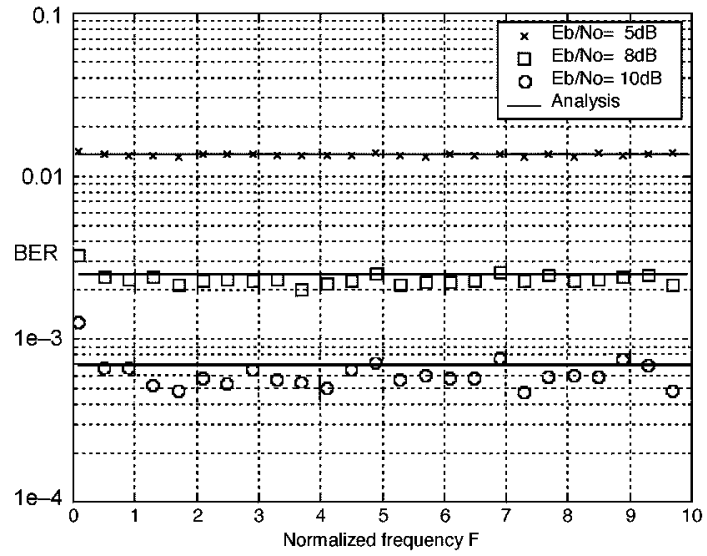

(c)

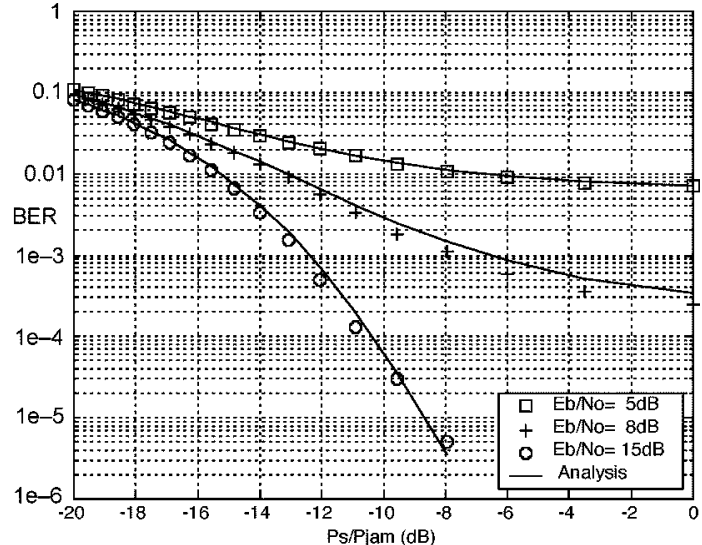

(b)

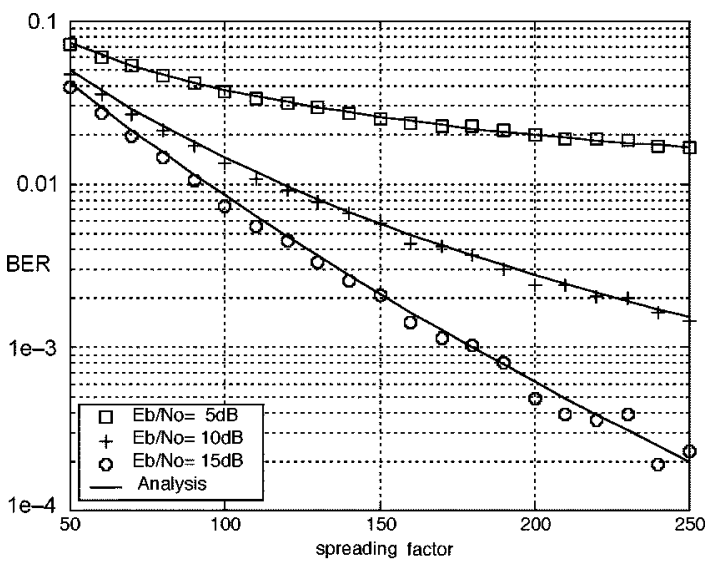

(d)

Fig. 4. BER of the coherent CSK system. Simulated BERs are plotted as points and analytical BERs plotted as lines. (a) BER versus average $E_{b} / N_{0}$. Jamming level $P_{s} / P_{\mathrm{jam}}$ is $-6.02,-9.54$ and $-12.04 \mathrm{~dB}$. Spreading factor is 200 . Normalized jamming frequency is 3.12735 . (b) BER versus $P_{s} / P_{\text {jam }}$. Average $E_{b} / N_{0}$ is 5, 8 , and $15 \mathrm{~dB}$. Spreading factor is 200 . Normalized jamming frequency is 3.12735 . (c) BER versus the jamming frequency. Average $E_{b} / N_{0}$ is 5,8 , and $10 \mathrm{~dB}$. Jamming level $P_{s} / P_{\mathrm{jam}}$ is $-9.54 \mathrm{~dB}$. Spreading factor is 200. (d) BER versus the spreading factor. Average $E_{b} / N_{0}$ is 5,10 , and $15 \mathrm{~dB}$. Jamming level $P_{s} / P_{\mathrm{jam}}$ is $-12.04 \mathrm{~dB}$.

Moreover, when the jamming power is zero, i.e., $P_{\mathrm{jam}}=0$, it can be readily shown that the bit error rate reduces to

$$
\begin{aligned}
& \operatorname{BER}_{\text {DCSK }} \mid\left(P_{\text {jam }}=0\right) \\
& =\frac{1}{2} \operatorname{erfc}\left(\frac{1}{\sqrt{\frac{2 \Lambda}{\beta P_{s}^{2}}+\frac{2 N_{0}}{\beta P_{s}}+\frac{N_{0}^{2}}{2 \beta P_{s}^{2}}}}\right) \\
& =\frac{1}{2} \operatorname{erfc}\left(\frac{1}{\sqrt{\left(\frac{E_{b}^{2}}{8 \beta \Lambda}\right)^{-1}+4\left(\frac{E_{b}}{N_{0}}\right)^{-1}+2 \beta\left(\frac{E_{b}}{N_{0}}\right)^{-2}}}\right) .
\end{aligned}
$$

Example: Consider the case where the logistic map (39) in Section III-A is used for generating the chaotic sequence. Putting (41) and (42) in (73) gives (77) as shown at the bottom of the page.

\section{COMPUTER SimUlations AND Discussions}

\section{A. Simulation Results}

In this subsection the anti-jamming performances of the coherent CSK system and the noncoherent DCSK system are studied by computer simulations. The logistic map described in Section III-A has been used to generate the chaotic sequences in both cases. In particular the following aspects of performance are relevant:

- BER versus the average ratio of the bit energy to noise spectral density $\left(E_{b} / N_{0}\right)$ in the presence of a jamming signal;

- BER versus the ratio of the signal power to the jamming signal power $\left(P_{s} / P_{\text {jam }}\right)$ for constant $E_{b} / N_{0}$;

- BER versus the normalized jamming frequency defined in (5);

- BER versus the spreading factor.

The relevant simulated BERs for the coherent CSK system are shown in Fig. 4, and that for the noncoherent DCSK system is shown in Fig. 5.

$$
\begin{aligned}
& \operatorname{BER}_{\mathrm{DCSK}}=\frac{1}{4} \int_{-1}^{+1} \operatorname{erfc}\left(\frac{\frac{\beta}{2}+\beta P_{\mathrm{jam}} \cos (\pi F)-\frac{P_{\mathrm{jam}} \sin (\pi F)}{\sin (\pi F / \beta)} W}{\sqrt{\frac{\beta}{4}+4 \beta P_{\mathrm{jam}} \cos ^{2}\left(\frac{\pi F}{2}\right)+\beta N_{0}+2 \beta P_{\mathrm{jam}} N_{0}+\frac{\beta N_{0}^{2}}{2}}}\right) \\
&\left.+\operatorname{erfc}\left(\frac{\frac{\beta}{2}-\beta P_{\mathrm{jam}} \cos (\pi F)+\frac{P_{\mathrm{jam}} \sin (\pi F)}{\sin (\pi F / \beta)} W}{\sqrt{\frac{\beta}{4}+4 \beta P_{\mathrm{jam}} \sin ^{2}\left(\frac{\pi F}{2}\right)+\beta N_{0}+2 \beta P_{\mathrm{jam}} N_{0}+\frac{\beta N_{0}^{2}}{2}}}\right)\right] \frac{1}{\pi \sqrt{1-W^{2}}} d W
\end{aligned}
$$




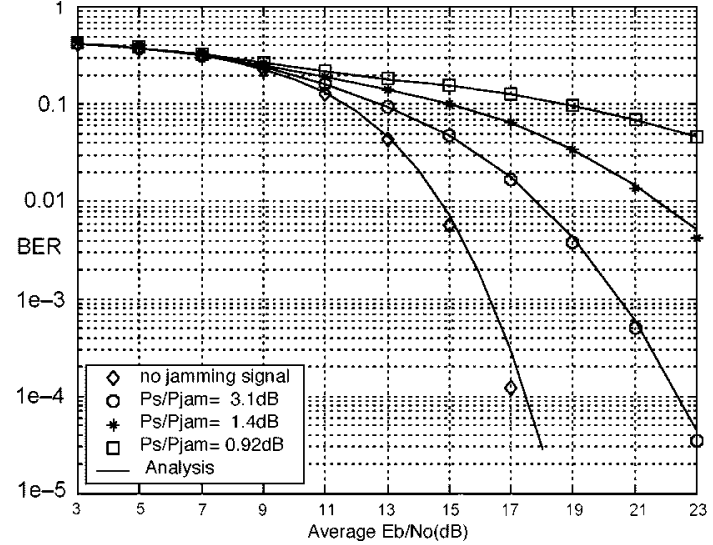

(a)

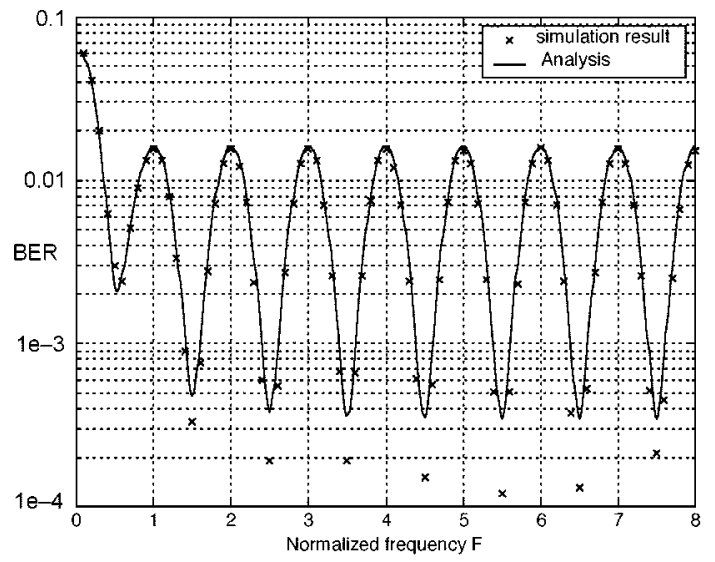

(c)

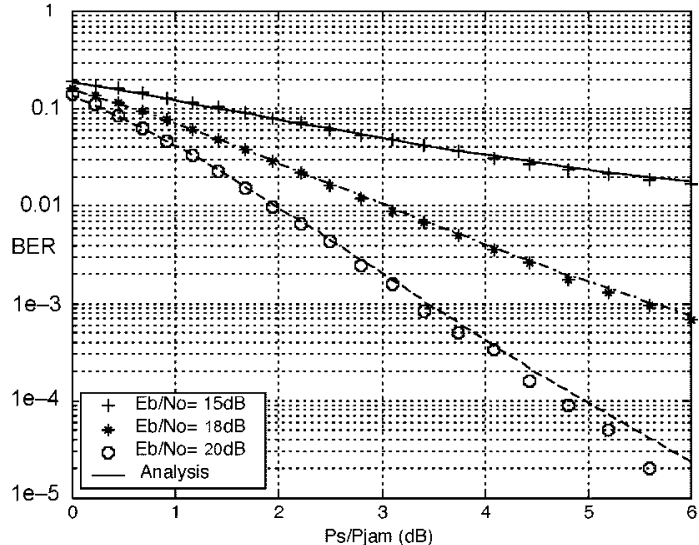

(b)

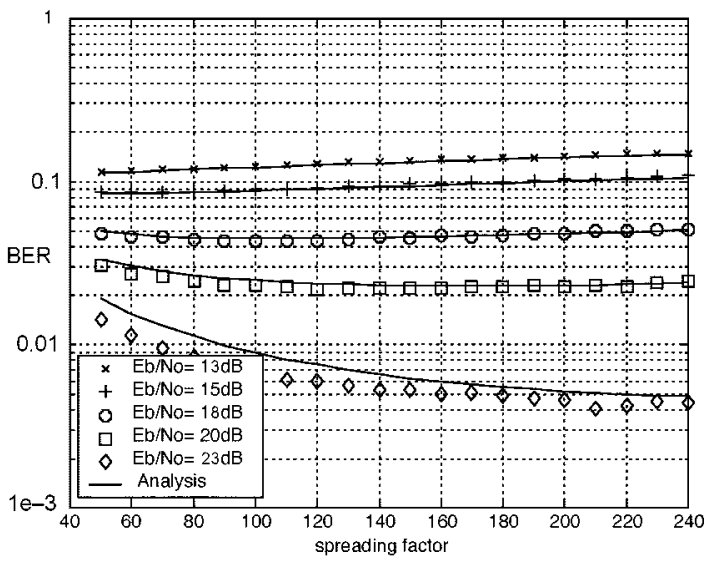

(d)

Fig. 5. BER of the noncoherent DCSK system. Simulated BERs are plotted as points and analytical BERs plotted as lines. (a) BER versus average $E_{b} / N_{0}$. Jamming level $P_{s} / P_{\text {jam }}$ is $0.92,1.4$ and $3.1 \mathrm{~dB}$. Spreading factor is 200. Normalized jamming frequency is 3.828734 . (b) BER versus $P_{s} / P_{\text {jam }}$. Average $E_{b} / N_{0}$ is 15,18 and $20 \mathrm{~dB}$. Spreading factor is 200 . Normalized jamming frequency is 3.828734 . (c) BER versus the normalized jamming frequency. Average $E_{b} / N_{0}$ is $18 \mathrm{~dB} . P_{s} / P_{\text {jam }}$ is $3.1 \mathrm{~dB}$. Spreading factor is 200 . (d) BER versus the spreading factor. Average $E_{b} / N_{0}$ is $13,15,18,20$ and $23 \mathrm{~dB}$. Jamming level $P_{s} / P_{\text {jam }}$ is $1.4 \mathrm{~dB}$. Normalized jamming frequency is 3.828734 .

In all cases, we plot also the analytical BERs obtained from the expressions derived in Sections III-A and III-B. From the figures, the consistency between the analytical BERs and the simulated BERs is clearly evidenced.

\section{B. Discussions}

Our analyses and simulations have consistently shown that the coherent CSK system generally performs better than the noncoherent DCSK system in the presence of sinusoidal jamming signals. On the other hand, it must be noted that the assumption a clean reference being available at the CSK receiver is still not fulfilled using the existing synchronization approaches. Several other interesting observations are worth noting.

1) As expected, the BER generally decreases (improves) as the average $E_{b} / N_{0}$ increases and the jamming power decreases.

2) For the coherent CSK system, the jamming frequency has little effect on the BER, whereas for the noncoherent DCSK system, BER experiences maxima and minima as the jamming frequency varies. In particular, the noncoherent DCSK system is most severely affected when the jamming frequency is an integer multiple of the bit frequency $\left(1 / T_{b}\right)$. This effect can be attributed to the inherent bit structure of DCSK which splits one bit duration into two slots. If the jamming frequency is an integer multiple of the bit frequency, then for each bit, the two slots will be superposed by two identical sinusoidal signal segments (may differ by a factor "-1"), giving a high correlation of the two slots, regardless of what digital message is sent. This strongly biases the correlator output and causes great error in the recovered message.

3) In general, a larger spreading factor improves the anti-jamming performance of the coherent CSK system, while no significant difference in performance is observed in the noncoherent DCSK system as the spreading factor varies. This effect can be explained as follows. In the coherent CSK system, higher the spreading factor, more accurate is the information provided by the correlator regarding the message identity, irrespective of the presence of a jamming signal. This is because the receiver is assumed to be able to regenerate a clean synchronized replica of the chaotic samples. However, for the noncoherent DCSK system, increasing the spreading factor does not necessarily improve performance because noise and/or jamming signal is equally admitted in both the reference and the information-bearing half bits.

\section{CONCLUSION}

Chaos-based communication has aroused considerable interest in the past few years both in physics and engineering research communities. Noise performance has been studied for many different types of systems. Rarely studied but certainly of interest is the antijamming capability of these systems. This paper attempts to fill this gap by presenting an analysis of the antijamming performance of two typical chaos-based digital communication systems. The specific systems studied are the coherent chaos-shift-keying system and the noncoherent differential 
chaos-shift-keying system. Closed-form analytical expressions for the bit error probabilities are obtained and compared with simulation results. From these results, useful design data for chaos-based digital communication systems can be obtained. Finally, it would be interesting to compare the antijamming capability of chaotic systems with traditional spread-spectrum communication systems, which will probably lead to further publications. From the authors' point of view, compared with traditional spread-spectrum systems which use coherent detection, noncoherent chaotic systems such as DCSK will be less favorable, whereas coherent CSK systems should provide comparable performance.

\section{APPENDIX}

\section{AUTOVARIANCE FUNCTION OF $\left\{x_{k}^{2}\right\}$ FOR THE LOGISTIC MAP}

The autovariance of $\left\{x_{k}^{2}\right\}$ is given by

$$
\operatorname{cov}\left[x_{j}^{2}, x_{k}^{2}\right]=\mathrm{E}\left[x_{j}^{2} x_{k}^{2}\right]-\mathrm{E}\left[x_{j}^{2}\right] \mathrm{E}\left[x_{k}^{2}\right]
$$

We consider the case where $j \neq k$. Without loss of generality, assume $k=j+n$ for some positive integer $n$

$$
\begin{aligned}
\mathrm{E}\left[x_{j}^{2} x_{k}^{2}\right] & =\int_{-\infty}^{\infty} \rho(x) x^{2}\left(g^{(n)}(x)\right)^{2} d x \\
& =\int_{-1}^{1} \frac{1}{\pi \sqrt{1-x^{2}}} x^{2}\left(g^{(n)}(x)\right)^{2} d x
\end{aligned}
$$

where $\rho(x)$ denotes the invariant probability density function of $x_{j} ; g^{(2)}(x)=g(g(x)), g^{(3)}(x)=g\left(g^{(2)}(x)\right), \ldots, g^{(n)}(x)=$ $g\left(g^{(n-1)}(x)\right)$ and $g(x)=1-2 x^{2}$ for the logistic map under study. Making the substitution $x=\cos \phi$, (79) becomes

$$
\begin{aligned}
\mathrm{E}\left[x_{j}^{2} x_{k}^{2}\right] & =\int_{\pi}^{0} \frac{1}{\pi \sin \phi} \cos ^{2} \phi\left(g^{(n)}(\cos \phi)\right)^{2}(-\sin \phi) d \phi \\
& =\frac{1}{\pi} \int_{0}^{\pi} \cos ^{2} \phi\left(g^{(n)}(\cos \phi)\right)^{2} d \phi .
\end{aligned}
$$

Applying the formula $1-2 \cos ^{2} v=-\cos 2 v$ to $g^{(i)}(\cos \phi)(i=$ $1,2, \ldots, n-1)$ repeatedly, we have

$$
\begin{aligned}
g(\cos \phi) & =1-2 \cos ^{2} \phi=-\cos 2 \phi \\
g^{(2)}(\cos \phi) & =g(g(\cos \phi))=g(-\cos 2 \phi) \\
& =1-2 \cos ^{2}(2 \phi)=-\cos \left(2^{2} \phi\right) \\
g^{(3)}(\cos \phi) & =g\left(g^{(2)}(\cos \phi)\right)=g\left(-\cos \left(2^{2} \phi\right)\right) \\
& =1-2 \cos ^{2}\left(2^{2} \phi\right)=-\cos \left(2^{3} \phi\right) \\
\vdots & =\vdots \\
g^{(n)}(\cos \phi) & =g\left(g^{(n-1)}(\cos \phi)\right)=g\left(-\cos \left(2^{n-1} \phi\right)\right) \\
& =1-2 \cos ^{2}\left(2^{n-1} \phi\right)=-\cos \left(2^{n} \phi\right)
\end{aligned}
$$

$$
\begin{aligned}
=\frac{1}{4 \pi}[\phi & +\frac{1}{2} \sin 2 \phi+\frac{1}{2^{n+1}} \sin \left(2^{n+1} \phi\right) \\
& +\frac{1}{2^{n+2}+4} \sin \left(\left(2^{n+1}+2\right) \phi\right) \\
& \left.+\frac{1}{2^{n+2}-4} \sin \left(\left(2^{n+1}-2\right) \phi\right)\right]_{0}^{\pi}=\frac{1}{4} .
\end{aligned}
$$

Similarly it is readily shown that

$$
\mathrm{E}\left[x_{j}^{2}\right]=\mathrm{E}\left[x_{k}^{2}\right]=\int_{-\infty}^{\infty} \rho(x) x^{2} d x=\int_{-1}^{1} \frac{1}{\pi \sqrt{1-x^{2}}} x^{2} d x=\frac{1}{2} .
$$

Putting (82) and (83) into (78), it is proved that the autovariance for $\left\{x_{k}^{2}\right\}$ is vanishing for the logistic map.

\section{REFERENCES}

[1] U. Parlitz, L. O. Chua, L. Kocarev, K. S. Halle, and A. Shang, "Transmission of digital signals by chaotic synchronization," Int. J. Bifurc. Chaos, vol. 2, pp. 973-977, 1992.

[2] C. W. Wu and L. O. Chua, "Transmission of digital signals by chaotic synchronization," Int. J. Bifurc. Chaos, vol. 3, no. 6, pp. 1619-1627, 1993.

[3] L. Kocarev and U. Parlitz, "General approach for chaotic synchronization with application to communication," Phys. Rev. Lett., vol. 74, pp. 5028 -5031, June 1995.

[4] H. Dedieu, M. P. Kennedy, and M. Hasler, "Chaos shift keying: Modulation and demodulation of a chaotic carrier using self-synchronizing Chua's circuit," IEEE Trans. Circuits Syst. II, vol. 40, pp. 634-642, Oct. 1993.

[5] M. Hasler, "Engineering chaos for secure communication," Phil. Trans. R. Soc. Lond., vol. 353, no. 1710, pp. 115-126, 1995.

[6] U. Feldmann, M. Hasler, and W. Schwarz, "Communication by chaotic signals: The inverse system approach," Int. J. Circuit Theory Appl., vol. 24, pp. 551-579, 1996.

[7] G. Kolumbán, M. P. Kennedy, and L. O. Chua, "The role of synchronization in digital communication using chaos-Part I: Fundamentals of digital communications," IEEE Trans. Circuits Syst. I, vol. 44, pp. 927-936, Oct. 1997.

[8] M. P. Kennedy, R. Rovatti, and G. Setti, Eds., Chaotic Electronics in Telecommunications. Boca Raton, FL: CRC Press, 2000.

[9] C. C. Chen and K. Yao, "Stochastic-calculus-based numerical evaluation and performance analysis of chaotic communication systems," IEEE Trans. Circuits Syst. I, vol. 47, pp. 1663-1672, Dec. 2000.

[10] G. Kolumbán, G. K. Vizvari, W. Schwarz, and A. Abel, "Differential chaos shift keying: A robust coding for chaos communication," in Proc. Int. Workshop on Nonl. Dynamics of Electron. Syst., Seville, Spain, 1996, pp. $87-92$

[11] G. Kolumbán, G. Kis, Z. Jáko, and M. P. Kennedy, "A robust modulation scheme for chaotic communications," IEICE Trans. Fundament. Electron., Commun., Comp. Sci., vol. E81-A, no. 9, pp. 1798-1802, 1998.

[12] M. Hasler and T. Schimming, "Chaos communication over noisy channels," Int. J. Bifurc. Chaos, vol. 10, pp. 719-735, 2000.

[13] J. G. Proakis and M. Salehi, Communications Systems Engineering. Englewood Cliffs: Prentice Hall, 1994.

[14] T. Kohda and A. Tsuneda, "Even and odd-correlation functions of chaotic Chebychew bit sequences for CDMA," in Proc. Int. Symp. on Spread Spectrum Technology and Applications, Oulu, Finland, 1994, pp. 391-395.

[15] I. S. Gradshteyn and I. W. Ryzhik, Tables of Integrals Series and Products. New York: Academic, 1965.

Substituting (81) into (80), we obtain

$$
\begin{aligned}
& \mathrm{E}\left[x_{j}^{2} x_{k}^{2}\right]= \frac{1}{\pi} \int_{0}^{\pi} \cos ^{2} \phi \cos ^{2}\left(2^{n} \phi\right) d \phi \\
&= \frac{1}{\pi} \int_{0}^{\pi}\left(\frac{1+\cos 2 \phi}{2}\right)\left(\frac{1+\cos \left(2^{n+1} \phi\right)}{2}\right) d \phi \\
&= \frac{1}{4 \pi} \int_{0}^{\pi}\left(1+\cos 2 \phi+\cos \left(2^{n+1} \phi\right)+\frac{1}{2} \cos \left(\left(2^{n+1}+2\right) \phi\right)\right. \\
&\left.+\frac{1}{2} \cos \left(\left(2^{n+1}-2\right) \phi\right)\right) d \phi
\end{aligned}
$$

